\title{
AÇÃO DO FUNGO CERATOCYSTIS FIMBRIATA ELL. \& HALST., ISOLADO DE DIVERSOS HOSPEDEIROS, SOBRE MUDAS DE CACAUEIRO $\left.{ }^{(1}\right)$
}

FAUSTO JOAQUIM CORAL, ODAIR ALVES BOVI, Seção de Plantas Tropicais, e IVAN JOSE ANTUNES RIBEIRO, Divisão de Horticultura, Instituto Agronômico.

\section{RESUMO}

Em condiçôes de casa de vegetação, foi verificada a patogenicidade de diferentes cepas de Ceratocystis fimbriata Ell. \& Halst. sobre mudas de cacaueiro (Theobroma cacao L.). As cepas foram obtidas dos seguintes hospedeiros: Cajanus cajans L. ; Acacia decurrens Willd; Crotalaria juncea $L$. e Mangifera indica $L$. Os cultivares de cacaueiro utilizados foram os seguintes: SCA $6 \mathrm{x}$ ICS 8; SCA 6 x UF 667; SCA $12 \times$ UF 677; SCA 12 x UF 667; IMC $67 \times$ UF 668; ICS $45 \times$ SCA 6; ICS $45 \times$ SCA 12; ICS $95 \times$ SCA 12 e UF 677 x SCA 6. Após 120 dias de observações, verificou-se que nenhuma das oepas de Ceratocystis inoculadas causou qualquer tipo de sintomas nas plantas de cacaueiro.

\section{INTRODUÇÃO}

Em muitos países produtores de cacau (Theobroma cacao L.), o fungo Ceratocystis fimbriata Ell. \& Halst. vem sendo mencionado como causador de severos danos à cultura (10).

No Brasil, ainda não se identificaram plantas de cacaueiro atacadas por esse patógeno, cuja ação já se faz sentir com grande

(1) Trabalho apresentado na 28. a Reunião Anual da Sociedaołe Brasileira para o Progresso da Ciência, realizada em Brasilia (DF), 7-14 de julho de 1976. Recebida para publicação a 4 de maio de 1983. 
intensidade (6) sobre outras culturas, tais como mangueira (Mangifera indica L.) e crotalária (Crotalaria juncea L.).

O estudo de inoculações cruzadas com o fungo Ceratocystis fimbriata não é recente. VIÉGAS (11) obteve resultados positivos quando, utilizando cepas isoladas de mangueira, inoculou plantas de crotalária, feijoeiro (Phaseolus vulgaris L.) e cafeeiro (Coffea arabica L.). MALAGUTI (4) causou sintomas da moléstia tanto em crotalária como em cafeeiro, inoculando-os com cepas isoladas de cacaueiro. Por sua vez, RIBEIRO \& CORAL (9), trabalhando com uma única cepa do patógeno isolada de mangueira, não conseguiram infectar mudas de cultivares híbridos de cacaueiro. Inoculaçōes cruzadas conduzidas por PONTIS (8) mostraram que cepas de Ceratocystis fimbriata do cafeeiro não eram patogênicas à batata-doce (Ipomoea batatas Lam.), ocorrendo o mesmo quando inoculou uma cepa isolada de batata-doce sobre o cafeeiro. BARBA-DONOSO (1), em Costa Rica, ao contrário de PONTIS (8), demonstrou que cepas isoladas de batata-doce foram patogênicas ao cafeeiro e cacaueiro. BARBA-DONOSO \& HANSEN (2), trabalhando com o patógeno isolado de cacaueiro, cafeeiro e batata-doce, conseguiram causar sintomas da moléstia em mudas de cacaueiro inoculadas, porém as cepas isoladas da batata-doce não foram patogênicas ao cafeeiro, confirmando as observaçōes de PONTIS (8). OLSON (7), trabalhando com batata-doce, verificou a ocorrência de dez cepas de Ceratocystis fimbriata que diferiram em patogenicidade, morfologia e compatibilidade. MEDEIROS (5), por outro lado, descreve a ocorrência de três raças fisiológicas do patógeno em crotalária.

Agora que o Estado de São Paulo se prepara para expandir sua área de cultivo com cacaueiros, empregando cultivares híbridos interclonais, torna-se desejável conhecer se entre a população do patógeno ocorrem biótipos capazes de infectar esse material.

\section{MATERIAL E METODOS}

Estudaram-se nove híbridos interclonais de cacaueiro inoculados com quinze diferentes cepas de Ceratocystis fimbriata isoladas dos seguintes hospedeiros: feijão-guandu (Cajanus cajan (L.) Millsp.) (umra cepa); acácia-negra (Acacia decurrens Willd (duas cepas); crotalária (Crotalaria juncea L.) (uma); mangueira (Mangifera indica L.) (onze). Os cultivares de cacaueiro utilizados foram os seguintes: SCA $6 \times$ ICS 8; SCA $6 \times$ UF 667; SCA $12 \times$ UF 677; SCA $12 \times$ UF 667; IMC 67 x UF 668; ICS $45 \times$ SCA 6; ICS $45 \times$ SCA 12; ICS $95 \times$ SCA 12 e UF 677 x SCA 6 . Todas as mudas, quando da implantação do ensaio, tinham dez meses de idade e haviam sido obtidas através de polinizações manuais controladas. O inóculo foi preparado, repicando-se as diferentes cepas do patógeno para meio de batata-dextrose-ágar e mantendo-as em estufa incubadora por vinte dias a $25^{\circ} \mathrm{C}$. As inoculações foram 
feitas com auxílio de um bisturi, com o qual se fazia ligeiro ferimento no caule dos cacaueiros, introduzindo-se aí pequena porção de micélio e esporos de Ceratocystis fimbriata. Como testemunha, utilizaram-se plantas de todos os cultivares estudados nas quais também se praticaram incisões com bisturi, cuidando-se, porém, que sobre os ferimentos não incidissem porções do patógeno. O ensaio foi mantido sob condições de casa de vegetação, verificando-se semanalmente o estado das plantas.

\section{RESULTADOS E DISCUSSÃo}

Após quatro meses de observações, as plantas nada revelaram de anormal, tendo os ferimentos cicatrizado e os tecidos internos apresentado coloração uniforme, tal qual as plantas testemunhas. ENRIQUEZ \& SORIA V. (3) mostraram que o clone IMC 67 é altamente resistente ao patógeno, enquanto SCA 6, SCA 12 e ICS 8 se apresentam moderadamente resistentes. Mostraram ainda que os clones ICS 95 e UF 667 são altamente suscetíveis ao fungo. Assim, como entre os materiais de cacaueiro testados existiam alguns cujos progenitores eram suscetíveis ou com resistência moderada, é de supor que os cultivares utilizados apresentassem diferentes níveis de resistência a Ceratocystis fimbriata, e a não-ocorrência de qualquer tipo de sintoma provou que as cepas utilizadas não thes foram patogênicas. Esses dados confirmam as observações de outros autores $(\mathbf{2}, \mathbf{8})$, que verificaram, em testes de inoculações cruzadas, que em alguns casos o patógeno mostrava uma tendência de especificidade ao hospedeiro.

\section{CONCLUSÃO}

Pode-se concluir desses resultados que o fungo Ceratocystis fimbriata isolado de feijão-guandu, acácia-negra, crotalária e mangueira não apresentou cepas patogênicas aos cultivares híbridos de cacaueiro utilizados.

\section{SUMMARY}

\section{ACTION ON COCOA PLANTS OF THE FUNGUS CERATOCYSTIS FIMBRIATA ELL. \& HALST. ISOLATED FROM DIFFERENT HOST PLANTS}

A greenhouse experiment was conducted to determine the pathogenicity of 15 different isolates of Ceratocystis fimbriata Ell. \& Halst. in cocoa (Theobroma cacao L.) plants. The isolates were obtained from Cajanus cajans L., Acacia decurrens Willd, Crotalaria juncea L. and Mangifera indica L. Potted cocoa plants (10 months old) representing nine different hybrids were used: SCA 6 × ICS 8 SCA $6 \times$ UF 667, SCA $12 \times$ UF 677, SCA $12 \times$ UF 667, IMC $67 \times$ UF 668, ICS $45 \times$ SCA 6 , ISC $45 \times$ SCA 12, ICS $95 \times$ SCA 12 and UF $677 \times$ SCA 6. Evaluation of damage was made visually at 120 days after inoculation. The results indicated no differences between control and inoculated plants. 


\section{REFERENCIAS BIBLIOGRÁFICAS}

1. BARBA-DONOSO, C. Estudio morfológico y pruebas de patogenicidad de varias cepas de Ceratocystis fimbriata Ell. \& Halst. In: Instituto Interamericano de Ciências Agricolas. Tesis de magister agriculturae de la Escuela para graduados 1947-1963: resúmenes analíticos. Turrialba, 1964. p.23. (Bibliotecologia y documentación, 3)

2. \& HANSEN, A. J. Pathogenicity test with different isolates of Ceratocystis fimbriata. Phytopathology, 52:2, 1962. (Abstracts)

3. ENRIQUEZ, G. A. \& SORIA V., J. Catalogo de cultivares de cacao. Turrialba. Centro de Enseñanza e Investigación, 1967. 300 fls.

4. MALAGUTI, G. Una podredumbre del talo de Crotalaria juncea causada por Ceratostomella fimbriata. Agronomia Tropical, 1(4):287-292, 1952.

5. MEDEIROS, A. G. Seleção de crotalária resistente a Ceratocystis fimbriata Ell. \& Halst. Fitopatologia, 2:29-34, 1967.

6. OLIVEIRA, F. T. G. Situação da cultura da mangueiræ no município de Jardinópolis. In: SIMPÓSIO SÔBRE A SECA DA MANGUEIRA, Campinas, 1966. Campinas, DATE, 1966. p.17-22. (Mimeografado)

7. OLSON, E. O. Genetics of Ceratostomella. I.Strains in Ceratostomella fimbriata Ell. \& Halst., Elliott from sweet potatoes. Phytophathology, 39:548-561, 1949.

8. PON'TIS, R. E. A canker disease of the coffee tree in Colombia and Venezuela. Phytopathology, 41:178-184, 1951.

9. RIBEIRO, I. J. A. \& CORAL, F. J. Estudo preliminar da ação do fungo Ceratocystis fimbriata Ell. \& Fialst., causador da seca da mangueira (M. indica L.), sobre cacaueiros (Theobroma cacao L.) Bragantia, Campinas, 27:LXXXVII-LXXXIX, 1968 (Nota, 20)

10. SAUNDERS, J. L. El complejo Xylebirus-Ceratocystis de cacao. Madison, Dep. Ent. Univ, Wisconsin, 1964. 15p. (Datilografado)

11. VIÉGAS, A. P. Seca da mangueira. Bragantia, Campinas, 19:163-182, 1960 